\title{
3-Dimensional High-Resolution Solar Spectro-Polarimetry
}

\author{
Reiner Volkmer \\ Universitäts-Sternwarte, Geismarlandstr. 11, D-37083 Göttingen, \\ Federal Republic of Germany
}

\begin{abstract}
In order to obtain information on the magnetic field on the sun in two spatial dimensions, a spectro-polarimeter of high spatial, spectral, and temporal resolution was built in the German Vacuum Tower Telescope (VTT) at the Observatorio del Teide/Tenerife. The two-dimensional spectrometer in the VTT, using a Universal Birefringent Filter (UBF) and a Fabry-Perot Interferometer (FPI) to obtain narrow-band filtergrams with a spectral resolution of the order of $3 \cdot 10^{5}$ (approx. 22 $\mathrm{m} \AA$ at $6303 \AA$ ) and a spatial resolution of $0.2 \mathrm{arcsec} / \mathrm{pixel}$ (Bendlin et al. 1992, Bendlin and Volkmer 1993), was extended to work as a spectropolarimeter for measuring Stokes-I and Stokes-V profiles.
\end{abstract}

\section{The two-dimensional spectro-polarimeter}

Figure 1 gives a schematic representation of the spectro-polarimeter. In front of the UBF, a polarization analyser consisting of a quarter-wave plate followed by two crossed calcites with their axes turned by $\pm 45^{\circ}$ with respect to that of the $\lambda / 4$-plate splits the incoming beam into one of right and one of left circular polarization (Stokes-(I+V), Stokes-(I-V)) before the spectral analysis is performed. The filter combination consisting of the UBF and FPI mentioned above decreases the spectral bandwidth, so that finally narrow-band images are obtained with a 12-bit Peltier-cooled slow-scan CCD. Scanning through a line with 40 wavelength positions $(\Delta \lambda \approx 10 \mathrm{~mA})$ for the profile and the adjacent continuum is accomplished within a few seconds by tuning only the FPI. By means of a beamsplitter and another CCD, white-light images of the same field of view are taken strictly simultaneously with the narrow-band images to have the possibility of correcting for image motion and seeing-induced distortions. $\mathbf{A} \lambda / 8$ plate is inserted in front of the polarimeter to compensate for the instrumental polarization of the coelostat system, the primary mirror, and some flat mirrors.

\section{Speciflcations of the spectro-polarimeter}

Spectral range: $4000 \AA$ to $7800 \AA$

Spectral resolution: $\frac{\lambda}{\Delta \lambda}=3 \cdot 10^{5} \quad$ (variable)

Finesse (FPI): 45

Free spectral range: $1 \AA$ at $6302 \AA$ (variable) 


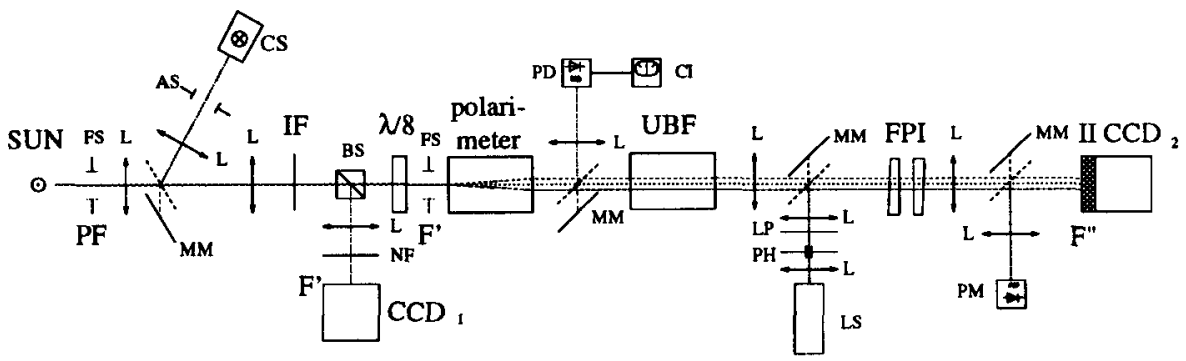

Figure 1. The two-dimensional spectro-polarimeter. Legend: FS: field stop, PF: prime focus, L: lens, MM: movable mirror, IF: interference filter, BS: beamsplitter cube, $\lambda / 8: \lambda / 8$-plate, $F^{\prime}, F^{\prime \prime}$ : foci, polarimeter: Stokes-V polarimeter, UBF: universal birefringent filter, FPI: Fabry-Perot interferometer, II: image intensifier, CCD: slow-scan CCD, AS: aperture stop, CS: continuum source, NF: neutral density filter, PD: photodiodes, CI: compensation indicator, LP: linear polarizer, PH: pinhole $(\varnothing=30 \mu \mathrm{m})$, LS: He-Ne laser source, PM: photomultiplier

Field of view: 25.6" $\times 17.8 " \quad(\max .57 .2 " \times 17.8 ")$

Beamsplitting caused by the polarimeter: $4 \mathrm{~mm}$ at F' $\left(18^{\prime \prime}\right.$ at $\left.\mathrm{CCD}_{2}\right)$

Spatial resolution: 0.4 arcsec ( $0.2 \mathrm{arcsec} / \mathrm{pixel}$, variable)

Image acquisition rate: $\approx 7.25$ images/s (field of view: $26 " \times 18$ ")

$\approx 3.5 \mathrm{images} / \mathrm{s}$ (maximum field of view)

\section{Observations}

Time series of white-light pictures and narrow-band images taken simultaneously were obtained in various lines from active and quiet regions. The Stokes-V polarimeter split every narrow-band image into one of right and one of left circular polarization (Stokes-(I+V), Stokes-(I-V)). For each scan, about 40 narrow-band filtergrams were obtained at different wavelength positions, yielding a line profile for every pixel in the field of view.

In the data reduction, seeing-induced effects were reduced via a destretching algorithm. Instrumental effects such as polarization and wavelength shifts were compensated for by different techniques. From each line profile, a set of parameters was derived, such as line-core and continuum intensity, velocity, Stokes-V signal, and line width. Thus, the temporal development of the observed solar features could be studied extensively.

Acknowledgments. This project has been supported by the Deutsche Forschungsgemeinschaft through grants Kn 152/9-1 and Kn 152/9-2.

\section{References}

Bendlin C., Volkmer R., Kneer F. 1992, A\&zA, 257, 817

Bendlin C., Volkmer R. 1993, A\&A, 278, 601 